\title{
ERRATUM 3
}

Article "Assessment of feedback for the teaching of nursing practice", with number of DOI: http://dx.doi.org/10.1590/0034-7167-2018-0539, published in the journal Revista Brasileira de Enfermagem, v72(3): 663-70, on page 663:

\section{Where it read:}

\section{ABSTRACT}

Objective: Construct and validate instrument content for nursing consultation in an adult chemotherapy outpatient clinic. Method: Methodological study composed of two stages: elaboration of the instrument and validation of content. A literary review of the dimensions of customer care was carried out in the light of Theory of Basic Human Needs Theory, culminating in two instruments: one for admission consultation and other for follow-up. The content was validated by the evaluation of listed experts based on the adapted Fehring's Validation Model. Results: In the first round, two items of the admission instrument and three items of follow-up required reformulation. In the second round, there was an increase in agreement rate: $11 \%$ in the instrument of admission and $10 \%$ in follow-up. Final Consideration: The instrument represents a guideline for the Nursing Process and future research, but it cannot be seen as a substitute for nurses' knowledge and clinical reasoning. Descriptors: Oncology Nursing; Nursing Process; Office Nursing; Validation Studies; Nursing Care.

\section{RESUMO}

Objetivo: Construir e validar conteúdo de instrumento para consulta de enfermagem em ambulatório de quimioterapia de adultos. Método: Estudo metodológico composto por duas etapas: Elaboração do instrumento e validação de conteúdo. Foi realizada revisão literária das dimensões do cuidado da clientela, à luz da Teoria das Necessidades Humanas Básicas, culminando em dois instrumentos: Um para consulta de admissão e outro de seguimento. O conteúdo foi validado pela avaliação de experts listados com base no Modelo de Validação de Fehring adaptado. Resultados: Na primeira rodada, dois itens do instrumento de admissão e três de seguimento necessitaram de reformulação. Na segunda rodada, houve aumento da taxa de concordância: $11 \%$ no instrumento de admissão e $10 \%$ de seguimento. Considerações Finais: $O$ instrumento representa um norteador do Processo de Enfermagem e de futuras pesquisas, mas não pode ser visto como um substituto do conhecimento e do raciocínio clínico do enfermeiro.

Descritores: Enfermagem Oncológica; Processo de Enfermagem; Enfermagem no Consultório; Estudos de Validação; Cuidados de Enfermagem.

\section{RESUMEN}

Objetivo: Construir y validar contenido de instrumento para consulta de enfermería en ambulatorio de quimioterapia de adultos. Método: Estudio metodológico compuesto por dos etapas: elaboración del instrumento y validación de contenido. Se realizó una revisión literaria de las dimensiones del cuidado de la clientela, a la luz de la Teoría de las Necesidades Humanas Básicas, culminando en dos instrumentos: uno para consulta de admisión y otro de seguimiento. El contenido fue validado por la evaluación de expertos listados con base en el Modelo de Validación de Fehring adaptado. Resultados: En la primera ronda, dos ítems del instrumento de admisión y tres de seguimiento necesitaban reformulación. En la segunda ronda, hubo aumento de la tasa de concordancia: $11 \%$ en el instrumento de admisión y $10 \%$ de seguimiento. Consideraciones Finales: El instrumento representa un orientador del proceso de enfermería y de futuras investigaciones, pero no puede ser visto como un sustituto del conocimiento y del raciocinio clínico del enfermero.

Descriptores: Enfermería Oncológica; Proceso de Enfermería; Enfermería de Consulta; Estudios de Validación; Atención de Enfermería. 


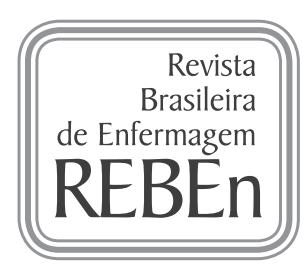

Read:

\begin{abstract}
Objective: to analyze the self-reported knowledge of professors in the use of feedback in the teaching of nursing practice; train professors to use this process; and evaluate the results of this training. Method: a qualitative study of research-action type. The focus group technique was used with seven collaborators of a nursing technical school. For content analysis, the Bardin framework was used and, for intervention, the Problematization Methodology supported by Pendleton's Rules. Results: most present in their curricula training to teach. It is pointed out that feedback is a simple and essential assessment tool, although universal discourse does not express the exact dimension of feedback potentialities. The intervention was effective to solidify feedback. Final considerations: this study shows that feedback should be disseminated and consolidated among the teaching staff of nursing technical education.
\end{abstract}

Descriptors: Educational Measurement; Health Evaluation; Learning; Feedback, Psychological; Education, Nursing.

\title{
RESUMO
}

Objetivo: analisar o conhecimento autorreferido de docentes na utilização do processo de feedback no ensino da prática de enfermagem; capacitar professores para utilização deste processo; e avaliar os resultados desta capacitação. Método: estudo qualitativo do tipo pesquisa-ação. Foi utilizada a técnica de grupo focal, com sete colaboradoras de uma escola técnica de enfermagem. Para análise do conteúdo, foi utilizado o referencial de Bardin e, para intervenção, a Metodologia da Problematização sustentada pelas Regras de Pendleton. Resultados: a maioria apresenta em seus currículos capacitação para educar. Aponta-se que o feedback é uma ferramenta simples e essencial de avaliação, embora o discurso universal não exprima a exata dimensão das potencialidades do processo. A intervenção foi efetiva para solidificação do processo de feedback. Considerações finais: este estudo mostra que o processo de feedback deve ser difundido e consolidado entre os docentes da área do ensino técnico de enfermagem.

Descritores: Avaliação Educacional; Avaliação em Saúde; Aprendizagem; Retroalimentação Psicológica; Educação em Enfermagem.

\section{RESUMEN}

Objetivo: analizar el conocimiento autorreferido de los docentes en la utilización del proceso feedback en la enseñanza de la práctica de enfermería; capacitar a estos profesores para utilizar este proceso y evaluar los resultados de esta capacitación. Método: estudio cualitativo del tipo investigación-acción. Se utilizó la técnica de grupo focal con siete colaboradoras de una escuela técnica de Enfermería. Para el análisis del contenido se utilizó el referencial de Bardin y, para la intervención, la metodología de problematización sostenida por las reglas de Pendlenton. Resultados: la mayoría presenta en sus currículos la capacitación para educar y apuntan que el feedback es una herramienta simple y esencial de evaluación, aunque el discurso universal no expresa la exacta dimensión de las potencialidades del proceso. La intervención fue efectiva para la solidificación del proceso de feedback. Consideraciones finales: este estudio muestra que el proceso de feedback debe ser difundido y consolidado entre los docentes del área de la enseñanza técnica de Enfermería.

Descriptores: Evaluación Educacional; Evaluación en Salud; Aprendizaje; Retroalimentación Psicológica; Educación en Enfermería. 